\title{
Dispersion Scan Frequency Resolved Optical Gating For Evaluation of Pulse Chirp Instability
}

\author{
Mariem Guesmi \\ Institute of Plasma Physics \\ Petra Veselá \\ Institute of Plasma Physics \\ Karel Žídek ( $\nabla$ zidek@ipp.cas.cz) \\ Institute of Plasma Physics
}

\section{Research Article}

Keywords: FROG, d-scan, D-FROG, NOPA

Posted Date: December 15th, 2020

DOI: https://doi.org/10.21203/rs.3.rs-125671/v1

License: (c) (i) This work is licensed under a Creative Commons Attribution 4.0 International License.

Read Full License 


\title{
Dispersion Scan Frequency Resolved Optical Gating For Evaluation of Pulse
}

\section{Chirp Instability}

\author{
M. Guesmi*, P. Veselá, K. Žídek*
}

Regional Center for Special Optics and Optoelectronic Systems (TOPTEC), Institute of Plasma Physics, Czech Academy of Science v.v.i., Za Slovankou 1782/3, 18200 Prague 8, Czech Republic

*Author to whom correspondence should be addressed. Email: guesmi@ipp.cas.cz

\section{zidek@ipp.cas.cz}

\section{Abstract}

The commonly used methods to characterize ultrafast laser pulses, such as frequency-resolved optical gating (FROG) and dispersion scan (d-scan), face problems when they are used on pulses varying within the acquisition time or laser beam. Such chirp variation can be identified by a discrepancy between the measured FROG and d-scan traces and their reconstructed counterparts. Nevertheless, quantification of the instability from the experimental data is a more complex task. In this work, we evaluate the precision of chirp instability quantification based on three different pulse characterization techniques. Two commonly used techniques FROG and d-scan are compared to a new method dispersion scan FROG (D-FROG) that combines the idea of dispersion scanning with the FROG method. We demonstrate the characterization of pulses generated from NOPA together with pulses processed by a 4f-pulse shaper without and with SLM-adjusted phase. In this paper, we validate the performance of the new method to estimate the chirp instability and, therefore, to improve the reconstruction of the measured results. Furthermore, we discuss the instability origin of each measurement case by using fast-scan autocorrelation traces. 
Introduction

Ultrafast laser pulses are used in many different application domains, including the pulsed laser deposition ${ }^{\underline{1}}$, femtochemistry, femtobiology ${ }^{2}$, or optical code division multiple access ${ }^{3}$. All the listed domains depend on reliable control and characterization of complex fs pulses, which can be carried out by a variety of methods. The simplest approach based on the autocorrelation technique provides only the minimum information about the shape of the pulse and a general waveform cannot be reliably recovered ${ }^{4}$. However, a range of other techniques makes it possible to recover even the complex pulse shapes. The most commonly used techniques are frequency-resolved optical gating (FROG) $)^{5,6,7}$, crosscorrelation frequency-resolved optical gating (XFROG) ${ }^{6}, 7$, spectral phase interferometry for direct electric-field reconstruction (SPIDER) $\stackrel{8}{ }$, multiphoton intrapulse interference phase scan (MIIPS) $)^{\underline{9}}$ and dispersion scan (d-scan) $\underline{10}$.

Many of these methods face a problem when they are applied to characterize a pulse shape varying within the beam or acquisition time. We denote this situation as a spatial and a dynamic chirp instability, respectively. In other words, the majority of standard pulse retrieval methods, including FROG, SPIDER and d-scan, assume that the pulse shape is constant throughout the acquisition time and the measured beam. However, this assumption can be corrupted by various phenomena, such as phase flickering induced during pulse shaping by the thermal fluctuations in spatial light modulators (SLMs) or by the effect of space-time coupling induced by the pulse shaping $\underline{14-16}$.

A prominent variation of the pulse electric field on a short time scale leads to the appearance of a narrow feature in the recorded nonlinear signal, which is called a "coherent artifact". This artifact can confuse interpretation of the autocorrelation or SPIDER measurements, including of the pulse length and temporal structure $\underline{12} \underline{13}$. On the contrary, the spatial and temporal chirp variations manifest themselves in the standard FROG setup as a discrepancy between the measured and the retrieved FROG traces ${ }^{6,11}$. Therefore, the chirp instability can be identified based on specific differences between 
the original and the retrieved FROG trace. Nevertheless, the instability quantification from the FROG experimental data represents a more challenging task that has not been yet addressed.

In this article, we study the use of three different methods to quantify chirp instability. Two commonly used techniques FROG and d-scan are compared to a new method, where we acquire FROG traces while we controllably vary the pulse dispersion by a known value of group delay dispersion (GDD) and third-order dispersion (TOD). We denote this method as dispersion scan FROG, shortly DFROG. The D-FROG dataset includes both the d-scan and a range of FROG traces. We demonstrate the use of the three different methods on a characterization of pulses (i) generated from a non-collinear optical parametric amplifier (NOPA); (ii) the same pulse passing through a 4f-pulse shaper without any modulation device; (iii), the same pulse passing through a 4f-pulse shaper with SLM-adjusted phase.

We fit the experimental data by a model, where we assume that the GDD values are not a single value, which would correspond to an ideal and stable pulse, but rather a distribution of the GDD values. The width of the distribution reflects the pulse instability. By scrutinizing the attained values, we show that the D-FROG method provides a means of careful evaluation of the laser pulse, which can reveal and quantify the instability of the pulse chirp on the order of tens of $\mathrm{fs}^{-2}$. We also demonstrate that even in the cases, where a single measured FROG trace can be well reproduced with a stable pulse, the D-FROG data provide a consistency check able to identify its instability.

\section{Experimental procedure}

The used experimental setup is depicted in Figure 1. We employed a fs laser system Pharos (Light Conversion) operated at $1028 \mathrm{~nm}$, which generated pulses $290 \mathrm{fs}$ long at $10 \mathrm{kHz}$ repetition rate, $100 \mu \mathrm{J} /$ pulse. A part of the output power ( $50 \mu \mathrm{J} /$ pulse) was converted by a non-collinear optical parametric amplifier (NOPA) N-3H Orpheus (Light Conversion) into a visible laser pulse at $640 \mathrm{~nm}$, spectral width FWHM $757 \mathrm{~cm}^{-1}$. We used a prism pulse compressor integrated into the NOPA to adjust pulse length and to vary the dispersion. The prism consisted of fused silica prisms separated by 775 $\mathrm{mm}$, where the prism P2 insertion was adjusted by a motorized stage to alter the pulse dispersion. It 
is worth noting that the measured pulse did not represent the best attainable pulse compression of the NOPA pulses. The presence of distinct features in the FROG traces was beneficial for the demonstration of the instability effect and dispersion scanning.

The pulse from NOPA was either directly characterized in a FROG setup, which is described later, or it was modified by a pulse shaper. We used a standard $4 \mathrm{f}$ pulse shaper - see Fig. $1 \mathrm{~b}$ employing grating $600 \mathrm{gr} / \mathrm{mm}$, which spectrally disperse the beam in the horizontal direction. The dispersed beam was collimated and focused in the vertical direction by a spherical mirror $(f=500 \mathrm{~mm})$ onto a spatial light modulator SLM-S640 (Jenoptik) placed in the Fourier plane. SLM was calibrated by using a procedure described in $\underline{\underline{17}}$. Consequently, symmetrically aligned mirrors were used to refocus the beam on the grating.

Finally, the temporal and spectral shape of the output pulse was measured by using the FROG setup. In this setup, the pulse was split with a pair of 50:50 beam splitters into two pulse replicas with an identical chirp. The delay between the pulses was varied by a motorized delay line (PIMag Linear Stage) and the pulses were focused on a $0.05 \mathrm{~mm}$ thick beta barium borate (BBO) crystal (Eksma Optics), where they generated a sum-frequency signal. Due to the non-collinearity of the incident pulses, the sum-frequency signal can be spatially separated by a pinhole and coupled with a lens into a fiber and analyzed by a spectrometer (AvaSpec-ULS4096CL-EVO). The laser spectrum was measured from a scattered beam on the pinholes (Ocean Optics Flame T). The second harmonic generation autocorrelation (SHG-AC) was acquired for the rapid scanning as a sum of the measured spectra. 

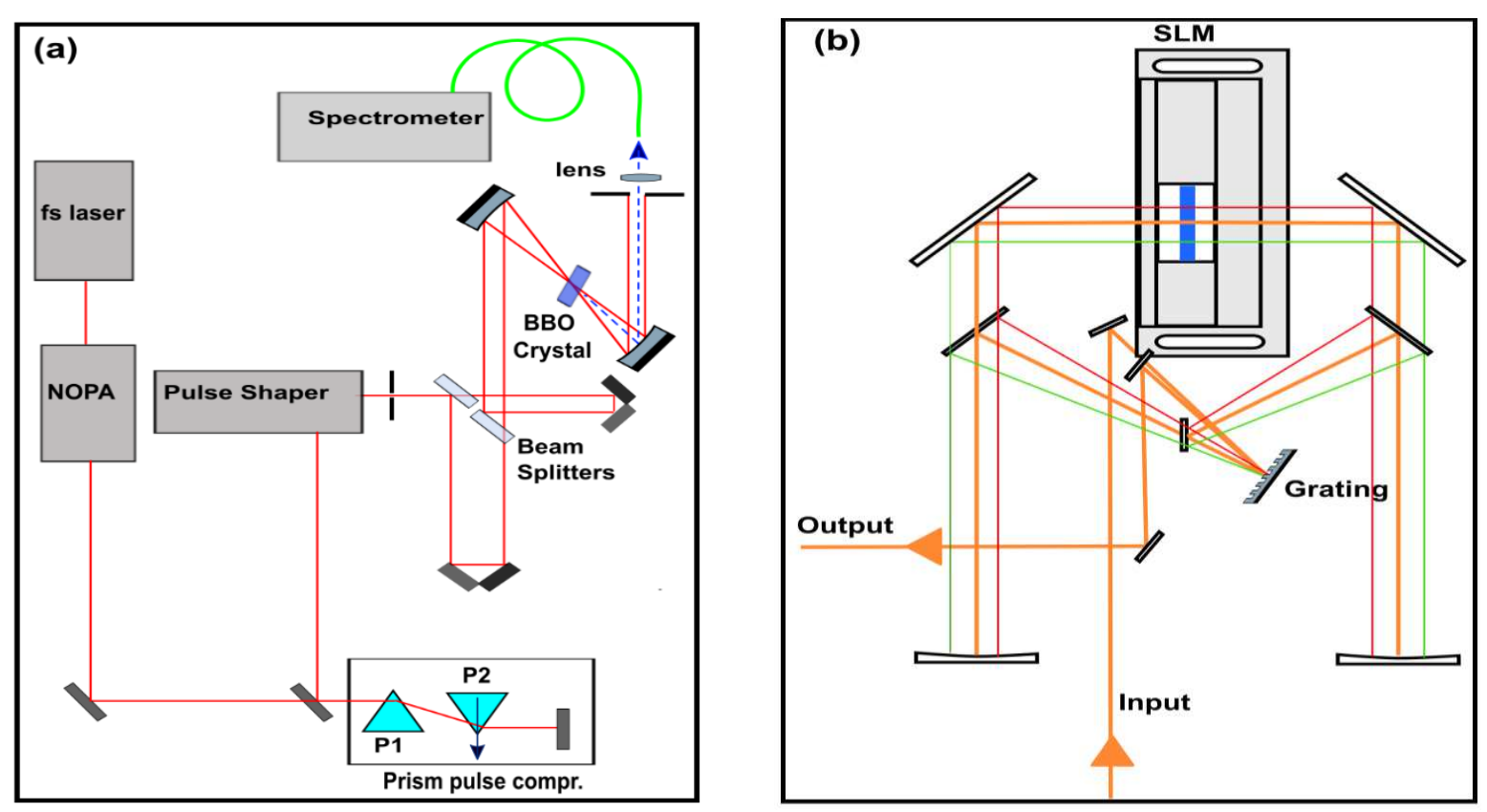

Figure 1. (a) Experimental setup for the D-FROG. (b) Pulse shaper in $4 \mathrm{f}$ geometry.

\section{Methods}

\section{Dispersion scan FROG}

Our setup is based on the second harmonic FROG, where the measured trace is given by:

$$
I_{F R O G}(\omega, \tau)=\left|\int E(t) E(t-\tau) \exp (-i \omega t) d t\right|^{2}
$$

where $\tau$ and $\omega$ are the pulse delay and the light frequency, respectively. $E(t)=\sqrt{I(t)} \exp (-i \Phi(t))$ is a complex amplitude of the pulse, where $I(t)$ is proportional to the light intensity and $\Phi(t))$ denotes the phase as function of time. The spectrum for $\tau=0$ corresponds to the SHG from a single pulse used in the d-scan measurements:

$$
I_{S H G}(\omega)=\left|\int E(t)^{2} \exp (-i \omega t) d t\right|^{2}
$$

Before the reconstruction, the SHG FROG trace is interpolated from the experimental data to form $N \times N$ matrix to reconstruct $N$ elements of intensity and phase vector. The FROG reconstruction is therefore an overdetermined system, which can be used to check the consistency of the results. 
Inconsistency leads to a difference between the measured $I_{F R O G}^{m}$ and the retrieved $I_{F R O G}^{r}$ FROG traces, which we evaluated as a discrepancy value $\delta$ :

$\delta_{F R O G}=\frac{1}{N^{2}} \sum_{\omega, \tau}\left(I_{F R O G}^{m}(\omega, \tau)-\mu \cdot I_{F R O G}^{r}\left(\omega_{i}, \tau\right)\right)^{2}$

Both experimental and theoretical traces are normalized to their maximum value and the parameter $\mu$ is optimized to obtaining the minimum discrepancy. The value of discrepancy $\delta$ is related to the commonly used error $G$ as $\delta=\mathrm{G}^{2}$.

A high value of the discrepancy indicates a problem in the measurement - for instance chirp instability in the system ${ }^{12}$. However, it is problematic to judge the actual origin of the issue - for instance to prove the presence of the chirp instability from the experimental data.

To overcome this problem, we employed an additional consistency check, where we controllably scan dispersion over a selected range to observe the phase change of the reconstructed pulse and the corresponding FROG trace. We scan the dispersion by varying position $p$ of a prism in the prism compressor (see experimental section). In the spectral domain, the electrical field is given by: $\tilde{E}(\omega)=\sqrt{S(\omega)} \exp (i \varphi(\omega))=\mathcal{F}\{E(t)\}$, where $\sqrt{S(\omega)}$ and $\varphi(\omega)$ are the spectral amplitude and phase, respectively. The phase $\varphi(\omega, \Delta p)$ for a certain change in prism insertion $\Delta p$ is given by a Taylor series as:

$\varphi(\omega, \Delta p)=\varphi_{C}+\varphi_{1}\left(\omega-\omega_{0}\right)+\frac{1}{2} G(\Delta p) \cdot\left(\omega-\omega_{0}\right)^{2}+\frac{1}{3} T(\Delta p) \cdot\left(\omega-\omega_{0}\right)^{3}$

where $\omega_{0}$ is the central frequency, $G$ represents the group delay dispersion (GDD) and $T$ is the $3 r d-$ order dispersion (TOD). $\varphi_{C}$ stands for phase of the pulse for $\Delta p=0$. The second term $\varphi_{1}$ causes the pulse translation in time and it can be ignored in the FROG calculation. At the same time, the higher dispersion terms can be neglected. 


\section{Chirp instability}

Under certain conditions - for instance, due to the fluctuating temperature of the SLM elements - it has been observed that pulse length rapidly varies in time $\underline{16} \underline{\underline{18}}$. In this case, FROG trace becomes a sum of FROG traces with a distribution of spectral chirp $\varphi(\omega, \Delta p)$. From the point of view of FROG measurements, the same case is also the pulse, where the chirp varies across the laser beam - for instance, due to space-time coupling induced in a $4 \mathrm{f}$ pulse shaper $\underline{\underline{14}}, \underline{15}$.

We can simulate the chirp instability by assuming that the resulting FROG trace arises as a sum of FROG traces from a set of pulses, where the quadratic chirp GDD is not a single value $G(\Delta p)$, but rather a distribution $D(g)$. We will use in the article a Gaussian distribution centered around the mean value $G(\Delta p)$ with the standard deviation $\sigma_{G}$ :

$D(g)=\frac{1}{\sqrt{2 \pi \sigma_{G}{ }^{2}}} \exp \left[-\frac{(g-G(\Delta p))^{2}}{2 \sigma_{G}{ }^{2}}\right]$

Nevertheless, a various set of models can be applied, depending on the expected chirp behavior. The field corresponding to the GDD value $g$ can be expressed as:

$E(t, g, \Delta p)=\mathcal{F}^{-1}\left\{\sqrt{S(\omega) \cdot} \cdot \exp \left[\varphi_{C}+\frac{1}{2} g \cdot\left(\omega-\omega_{0}\right)^{2}+\frac{1}{3} T(\Delta p) \cdot\left(\omega-\omega_{0}\right)^{3}\right]\right\}$,

which allows us to calculate the D-FROG signal $I_{D F}$ from an instable pulse as a function of prism insertion:

$I_{D F}(\omega, \tau, \Delta p)=\frac{1}{\sqrt{2 \pi \sigma_{G}}} \int \exp \left[-\frac{(g-G(\Delta p))^{2}}{2 \sigma_{G}{ }^{2}}\right]\left|\int E(t, g, \Delta p) E(t-\tau, g, \Delta p) \exp (-i \omega t) d t\right|^{2} d g$

By using Equation (7), we can extract the d-scan signal $I_{D S}$ by setting $\tau=0$, ie.

$I_{D S}(\omega, \Delta p)=\frac{1}{\sqrt{2 \pi \sigma_{G}}} \int \exp \left[-\frac{(g-G(\Delta p))^{2}}{2 \sigma_{G}{ }^{2}}\right]\left|\int E(t, g, \Delta p)^{2} \exp (-i \omega t) d t\right|^{2} d g$

To theoretically calculate the D-FROG, FROG and d-scan signal, we need to determine the GDD and TOD change with prism insertion $G(\Delta p)$ and $T(\Delta p)$. These can be attained by using the approach 
introduced as a self-calibrating $d$-scan $\underline{\underline{19}}, \underline{\underline{20}}$. The spectral intensity shape $S(\omega)$ was determined based on the laser spectrum. Finally, the spectral phase $\varphi_{C}$ for the reference prism insertion $\Delta p=0$ was estimated by a standard FROG trace reconstruction (described in detail later). Therefore, we could use Equations (7) and (8) to fit a single unknown parameter $\sigma_{G}$ from FROG, D-FROG and d-scan datasets. As a measure of the fit quality, we have also introduced discrepancies of the D-FROG and d-scan data analogously to Equation (3):

$$
\begin{aligned}
& \delta_{D F}=\frac{1}{\mathcal{N}_{i} N^{2}} \sum_{\omega, \tau}\left(I_{D F}^{m}(\omega, \tau, \Delta p)-\mu . I_{D F}^{r}\left(\omega_{i}, \tau, \Delta p\right)\right)^{2} \\
& \delta_{D S}=\frac{1}{\mathcal{N}_{i} N} \sum_{\omega, \tau}\left(I_{D S}^{m}(\omega, \tau)-\mu . I_{D S}^{r}\left(\omega_{i}\right)\right)^{2}
\end{aligned}
$$

In this case, we use an additional factor $\mathcal{N}_{i}$, which indicates the number of scanned prism insertion positions.

\section{Results and discussion}

We used three different methods: FROG, D-FROG, and d-scan to study and quantify the chirp instability. We will first introduce our methods on a simple case, where we directly measured a pulse generated from NOPA, which bypassed the pulse shaper.

In Figure 2, we depict the measured (upper left panel) and the retrieved (upper right panel) FROG traces from the compressed NOPA pulse. The traces reflect electric field amplitude, i.e. $\sqrt{\left|I_{\text {FROG }}(\omega, \tau)\right|}$, which is used in the ptychographic reconstruction procedure by Sidorenko et al.. $\underline{21}$. The pulse reconstruction provided us with the temporal and spectral intensities and phases of the pulse depicted in the lower panels. The step of FROG trace reconstruction was used in all measurements to estimate the spectral phase of the pulse $\varphi_{C}$ for the reference prism insertion $\Delta p=0$. 

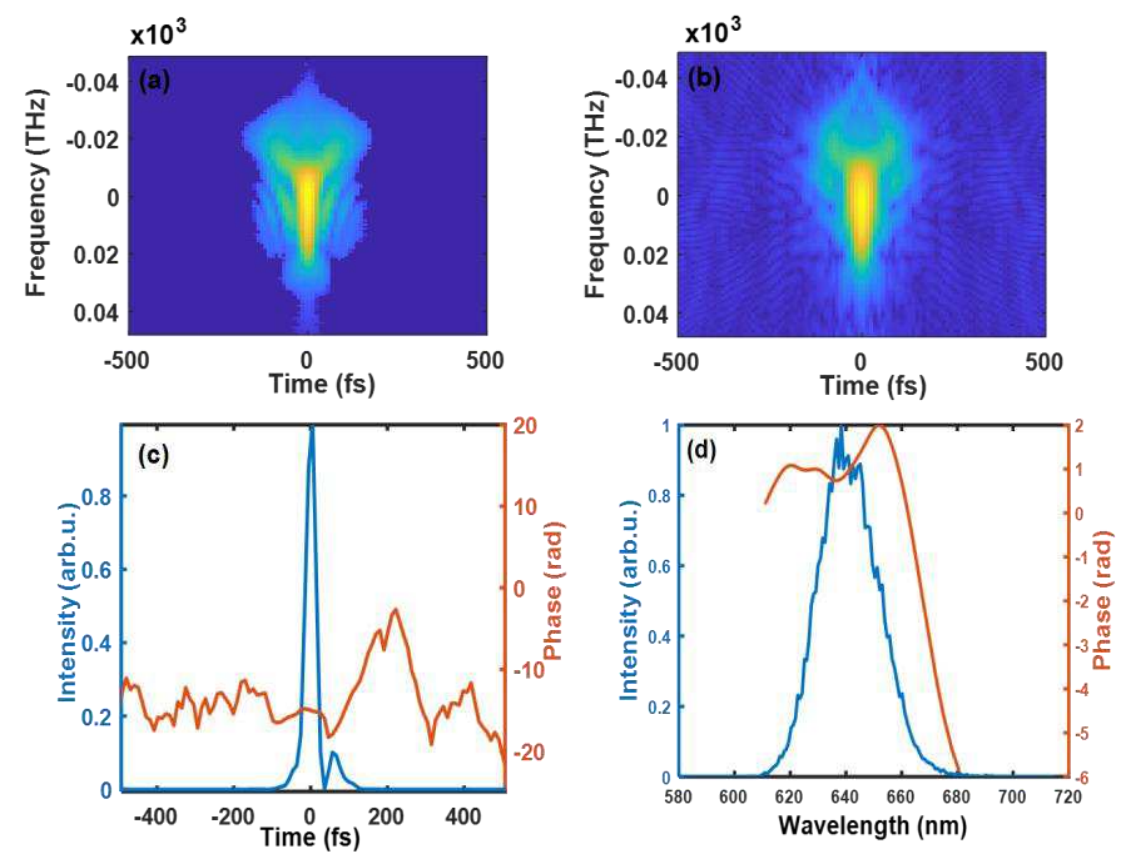

Figure 2. Measured (a) and Retrieved (b) FROG traces from a compressed NOPA pulse. (c) Retrieved temporal intensity profiles and phases. (d) Retrieved spectral intensity profiles and phases. $N=156$, $d t=1.032 \mathrm{fs}$. The retrieved fields with rms widths $16.75 \mathrm{fs}$.

The D-FROG and d-scan datasets were attained by scanning the prism insertion and FROG trace acquisition for each of the positions - see upper panels in Figure 3 (a) - and d-scan trace depicted in Figure 3 (b) - upper panel.

To retrieve the theoretical traces, we needed to find the dispersion change with the prism insertion, which we assumed to change linearly: $G(\Delta p) \propto \Delta p$ and $T(\Delta p) \propto \Delta p$. The values were extracted by using the self-calibration d-scan approach, where we fitted the d-scan experimental trace by using Equation (2). We set the FROG-reconstructed pulse as an initial guess, while the $G(\Delta p)$ and $T(\Delta p)$ scaling was left as a free fitting parameter. We extracted the values of $G(\Delta p) / \Delta p=500 \mathrm{fs}^{-2} / \mathrm{mm}$ and $T(\Delta p) / \Delta p=10 \mathrm{fs}^{-3} / \mathrm{mm}$

This allowed us to calculate the FROG, D-FROG and d-scan traces, which we first evaluated from Equations (1) and (2) for an ideal stable pulse. The difference between each measured and 
calculated trace is depicted in the panel below with a different color scheme, revealing minor discrepancies in the shapes. It is worth stressing that the agreement in 17 FROG traces with very different shapes is achieved by using a single reconstructed spectral phase $\varphi_{C}$ and two values of $G(\Delta p)$ and $T(\Delta p)$.

The subtle discrepancies between the measured and recovered traces, which are listed in Table 1 for all experiments, are a sign of the low chirp instability. This is also confirmed by the $d$-scan trace in Figure 3, which is not smeared along the prism insertion axis, as it would be expected for the unstable chirp.

NOPA: stable pulse

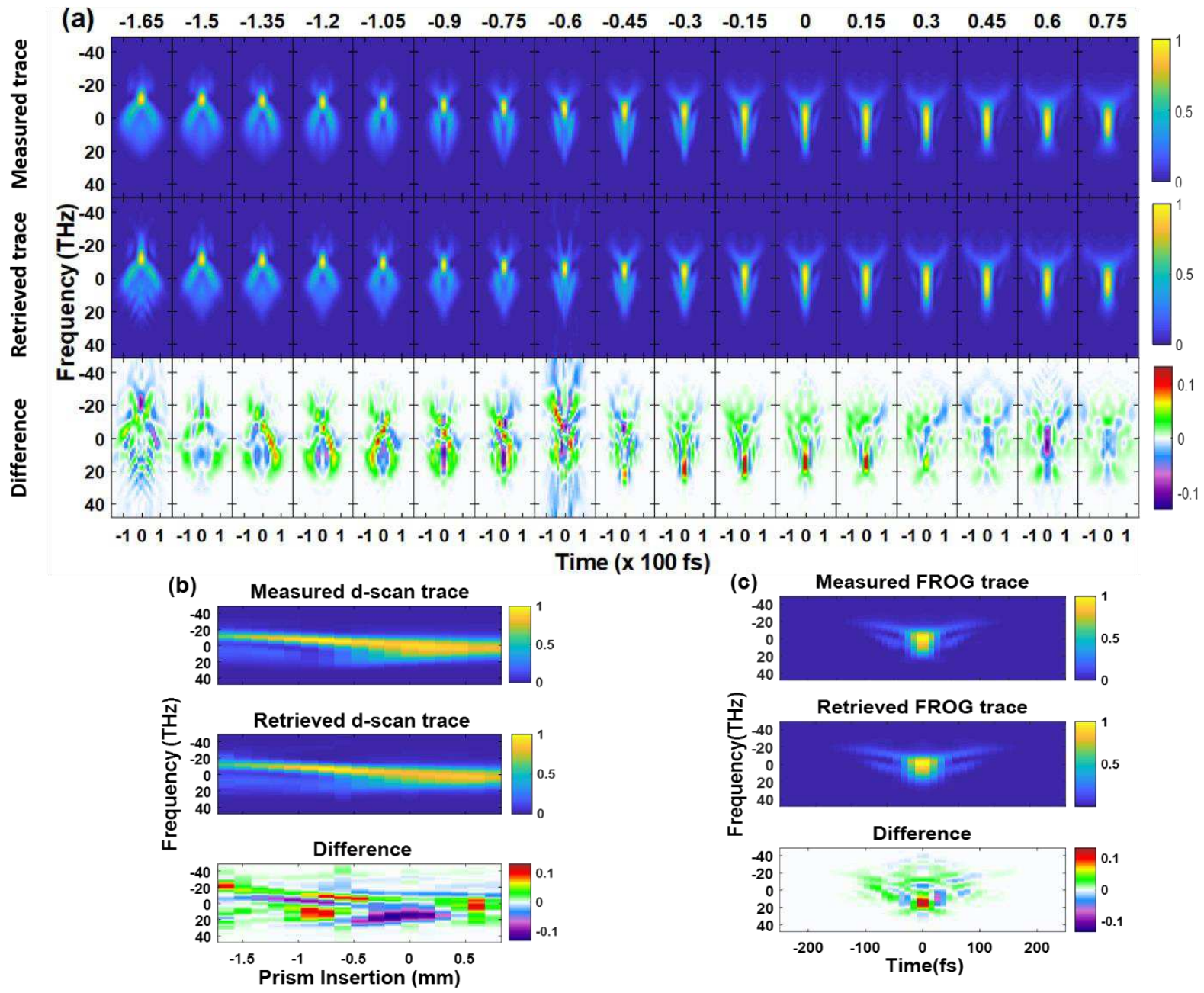


Figure 3. Measured and Retrieved traces of an ideal stable pulse, generated from NOPA, and their differences. (a) D-FROG traces (the traces are acquired for different prism insertion positions from -1.65 to 0.75 , as mentioned above the panels) (b) d-scan trace (c) FROG trace of a compressed pulse. The traces are retrieved using Eq (1) and (2), respectively, where $N=156$ and $d t=1.032 f s$.

To study and quantify this chirp instability, we fitted the FROG, D-FROG and d-scan traces from Equations (7) and (8) by leaving the chirp distribution width $\sigma_{G}$ as a free fitting parameter. The retrieved D-FROG and FROG traces are shown in Figure 4 (a) and Figure 4 (c), where their retrieved standard deviations $\sigma_{G}$ are equal to 30 and $0 \mathrm{fs}^{-2}$, respectively. Despite the pulse being almost ideally stable, the chirp instability fitting improves the retrieved traces and lowers the discrepancy values (see Table1). The d-scan datasets were depicted in Figure 4 (b) - upper panel.

NOPA: unstable pulse




Figure 4. Measured and Retrieved traces of an unstable pulse, generated from NOPA, and their differences. (a) D-FROG traces (the traces are acquired for different prism insertion positions from -1.65 to 0.75 , as mentioned above the panels) (b) d-scan trace (c) FROG trace of a compressed pulse. The traces are retrieved using Eq (7) and (8), respectively, where $N=156$ and $d t=1.032 f s$.

Since the pulses generated from NOPA are not expected to show prominent chirp instability, we extend our methods on a characterization of pulses generated from NOPA and processed with a 4f-pulse shaper and without any modulation device. The measured DFROG, $d$-scan, and FROG traces are depicted in Figure 5, where they are compared with calculated ideal traces for a stable pulse by using Equations (1) and (2). A significant difference between the measured and calculated traces compare Figure $5(a)$ and $(b)$ - can be spotted especially for the negative part of the frequency axis, where the experimental data is clearly smeared along the delay axis. This indicates a significant chirp instability. Analogously, the discrepancy can be spotted in the d-scan traces - see the prominent peak for the negative $\Delta p$ for frequency around $-100 \mathrm{THz}$, which is smeared in the experimental data. It is also worth noting that the difference for some particular FROG traces - for instance $\Delta p=0$ - can be negligible and it can be easily overlooked. 

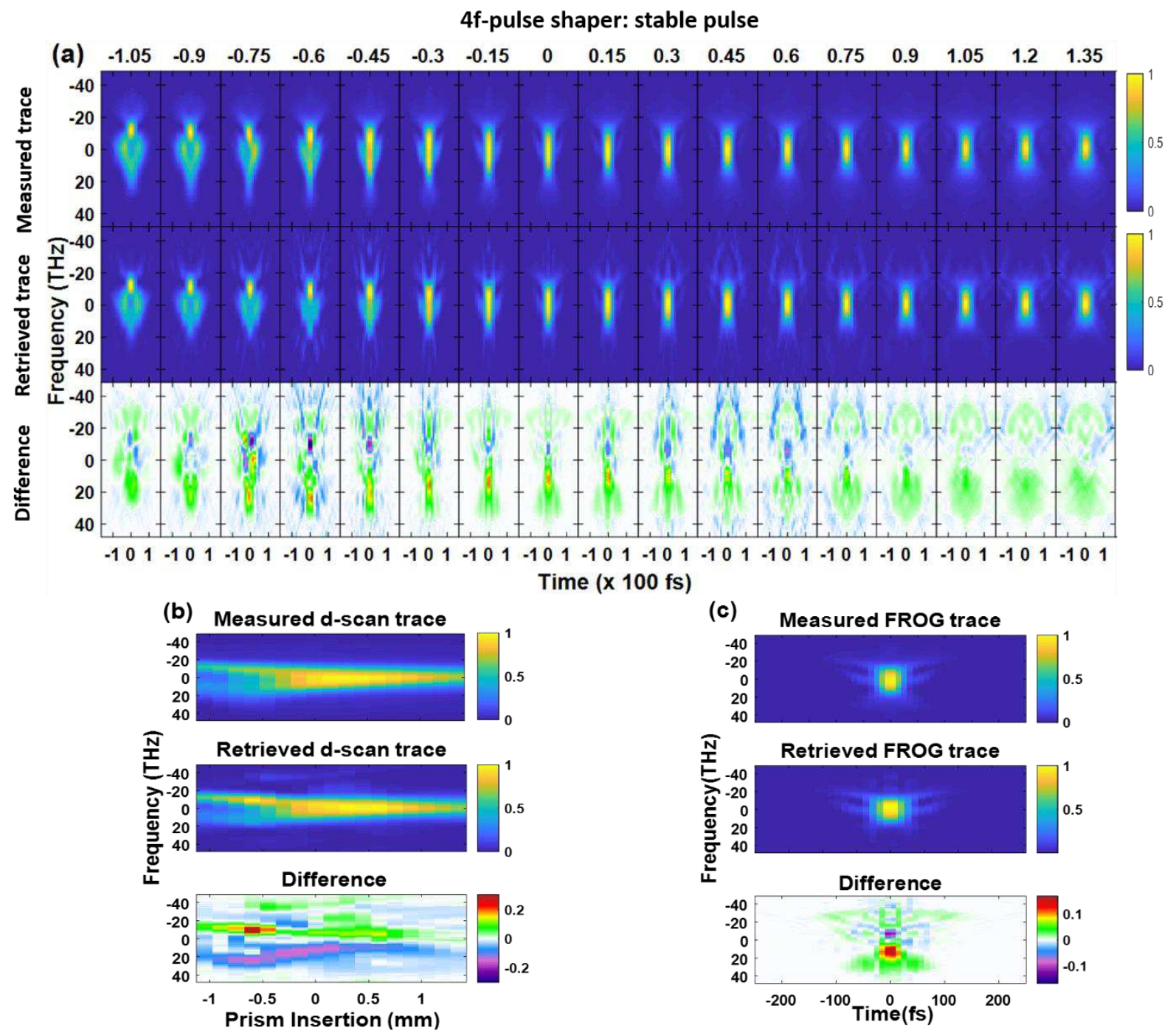

Figure 5. Measured and Retrieved traces of an ideal stable pulse, generated from NOPA and measured with a 4f-pulse shaper, and their differences. (a) D-FROG traces (the traces are acquired for different prism insertion positions from -1.05 to 1.35 , as mentioned above the panels) (b) d-scan trace (c) FROG trace of a compressed pulse. The traces are retrieved using Eq (1) and (2), respectively, where $N=156$ and $d t=1.032 \mathrm{fs}$.

To quantify this instability, we fitted the D-FROG traces by using Equations (7) and (8) and the phase extracted from the FROG experiment at $\Delta p=0$, analogously to the previous case -- see Figure 6 . We observed that the fitted value of chirp instability, which reproduces best the experimental D-FROG 
data, reached $110 \mathrm{fs}^{-2}$. When we focused only on the d-scan trace in Figure 6 (b) and optimized the chirp instability for the lowest discrepancy, we also reached the same value of $110 \mathrm{fs}^{-2}$. However, when we fit the chirp instability by using a single FROG trace (at $\Delta p=0$ ), we derive a significantly lower value $\left(40 \mathrm{fs}^{-2}\right)$. This is an expected result since the FROG trace is used for the phase reconstruction and the retrieved phase can partly compensate for the chirp instability.

Therefore, we tested the optimization of $\sigma_{G}$ value on three different spectral phases, where two were extracted from the FROG data (ptychograhic reconstruction) and one derived from d-scan pulse retrieval, which followed the procedure of $\operatorname{Ref}^{20}$. All three phases are compared in Figure $7 \mathrm{a}$. The red line in Figure 7(a) corresponds to the spectral phase employed in the previous reconstruction. Note that the phases differ substantially only in the parts, where the laser spectrum is below $5 \%$ of the peak intensity.

We studied the experimental-theoretical trace discrepancy as a function of the chirp instability $\sigma_{G}$ for each characterization method (FROG, d-scan and D-FROG)- see Figure 7(b). This allowed us to evaluate their sensitivity on the $\sigma_{G}$ value and on the varying pulse phase. We calculated the discrepancies for different chirp instability for all three phases (Figure 7 b), where each panel corresponds to one curve in Figure 7a. For the sake of better comparison, the discrepancy data were normalized on their lowest value. 
4f-pulse shaper: unstable pulse

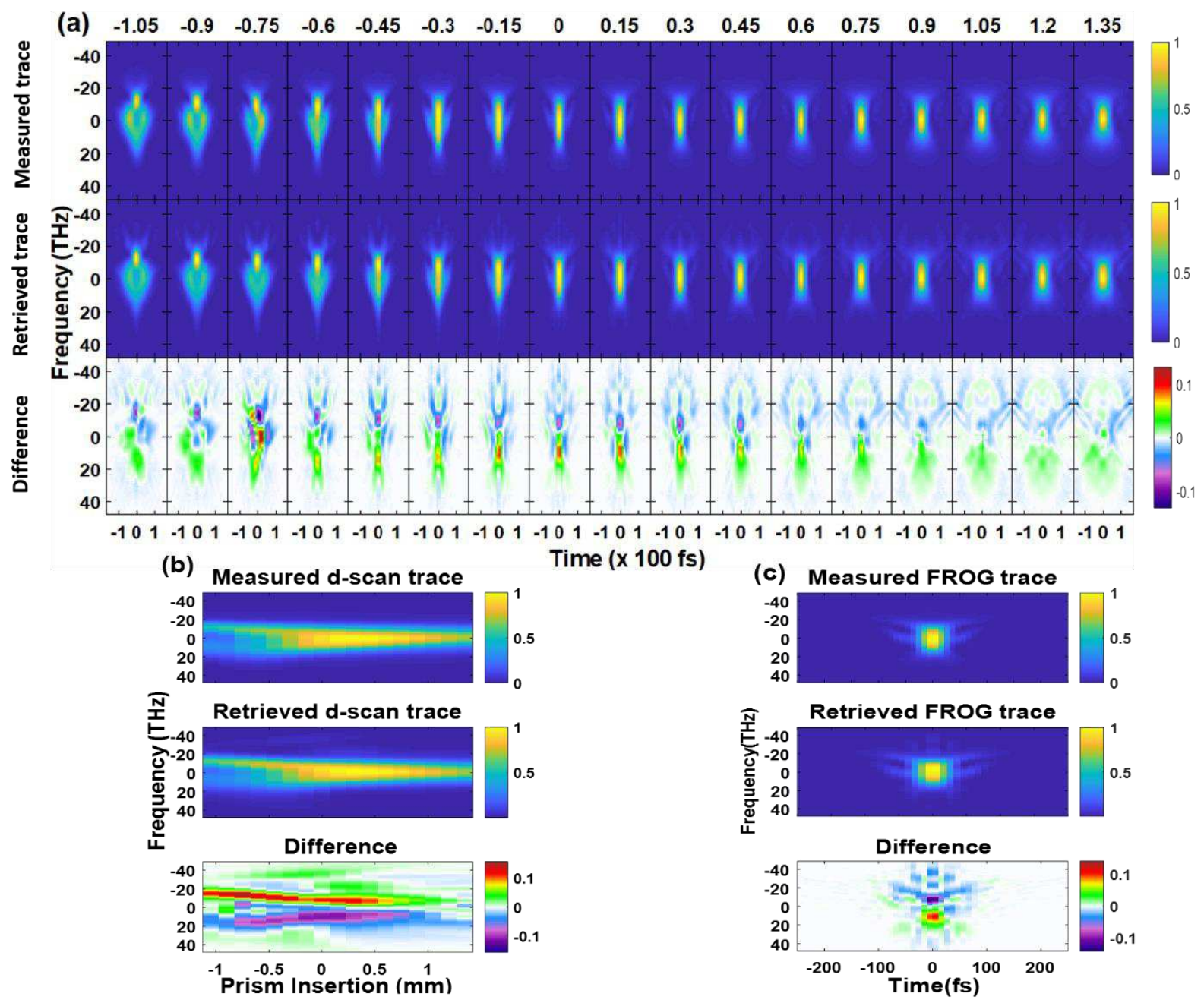

Figure 6. Measured and Retrieved traces of an unstable pulse, generated from NOPA and measured with a 4f-pulse shaper, and their differences. (a) D-FROG traces (the traces are acquired for different prism insertion positions from -1.05 to 1.35 , as mentioned above the panels) (b) d-scan trace (c) FROG trace of a compressed pulse. The traces are retrieved using Eq (7) and (8), respectively, where $N=156$ and $d t=1.032 f s$. 
Table 1. the discrepancy $\delta$ and chirp instability $\sigma_{G}$ values estimated using the FROG, D-FROG and dscan methods of the pulses generated from a NOPA then measured through a $4 \mathrm{f}$-pulse shaper without and with SLM-adjusted phase. Stable case corresponds to the value $\sigma_{G}=0$.

\begin{tabular}{|c|c|c|c|c|c|c|c|c|c|}
\hline Methods & \multicolumn{3}{|c|}{ FROG (compressed pulse) } & \multicolumn{3}{|c|}{ D-FROG } & \multicolumn{3}{|c|}{ d-scan } \\
\hline Pulses & stable & \multicolumn{2}{|c|}{ unstable } & stable & \multicolumn{2}{|c|}{ Unstable } & stable & \multicolumn{2}{|c|}{ unstable } \\
\hline Values & $\begin{array}{c}\delta \\
\left(10^{-5}\right)\end{array}$ & $\begin{array}{c}\sigma_{G} \\
\left(\mathrm{fs}^{-2}\right)\end{array}$ & $\begin{array}{c}\delta \\
\left(10^{-5}\right)\end{array}$ & $\begin{array}{c}\delta \\
\left(10^{-5}\right)\end{array}$ & $\begin{array}{c}\sigma_{G} \\
\left(\mathrm{fs}^{-2}\right)\end{array}$ & $\begin{array}{c}\delta \\
\left(10^{-5}\right)\end{array}$ & $\begin{array}{c}\delta \\
\left(10^{-3}\right)\end{array}$ & $\begin{array}{c}\sigma_{G} \\
\left(\mathrm{fs}^{-2}\right)\end{array}$ & $\begin{array}{c}\delta \\
\left(10^{-3}\right)\end{array}$ \\
\hline NOPA & 3.3 & 0 & 1.99 & 4.2 & 30 & 3.94 & 1.06 & 30 & 1.19 \\
\hline Pulse & \multirow{3}{*}{8.16} & 40 & 4.61 & \multirow{3}{*}{9.08} & 110 & 4.19 & \multirow{3}{*}{2.92} & 110 & 1.52 \\
\hline \multirow[t]{2}{*}{ Shaper } & & 200 & 9.34 & & 200 & 5.12 & & 200 & 1.90 \\
\hline & & 300 & 15.60 & & 300 & 7.97 & & 300 & 3.15 \\
\hline SLM & 16.09 & 220 & 13.12 & 20.85 & 240 & 11.59 & 4.91 & 240 & 2.63 \\
\hline
\end{tabular}


(a)

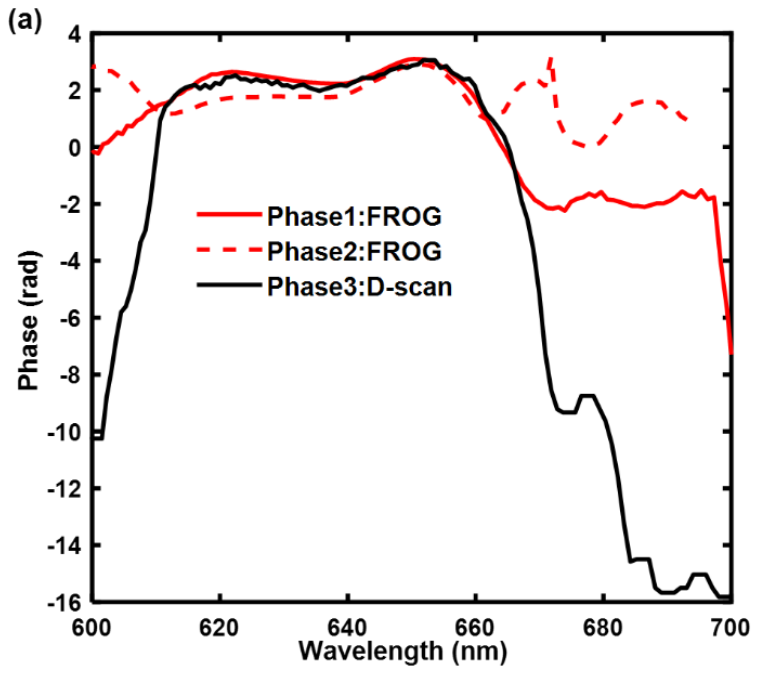

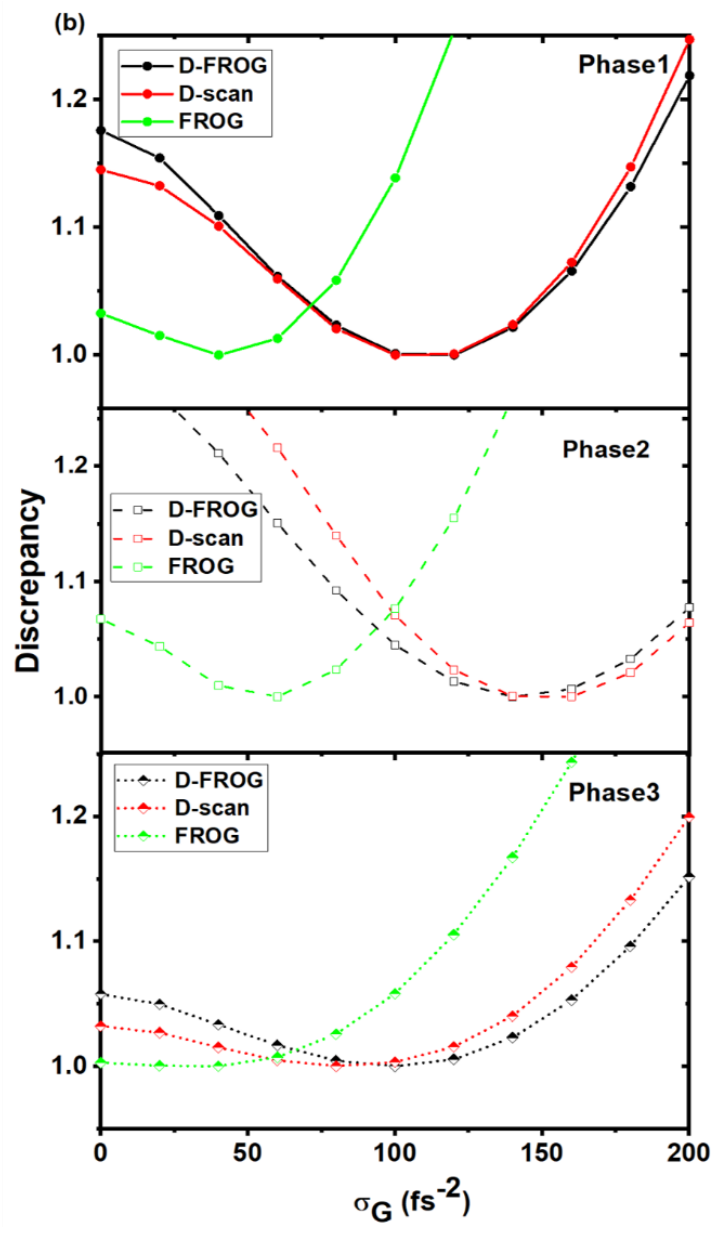

Figure 7. (a) Spectral phases, retrieved using FROG and d-scan methods, of a pulse generated from a NOPA and measured through a 4f-pulse shaper without any modulation device. (b) Discrepancy versus the chirp instability $\sigma_{G}$, using three different phases.

By comparing the discrepancy versus the chirp instability $\sigma_{G}$ for the different methods FROG, D-FROG and d-scan (see Figure 7(b)), we conclude that while the FROG method (green lines) can reveal the chirp instability and provide a consistent value, it tends to underestimate the chirp instability. On the other hand, the d-scan method (red lines) seems more reliable to detect the chirp instability than FROG. However, when we used the d-scan for the phase retrieval, the method partly compensated for the chirp instability in the pulse shape (see Figure 7b, lowest panel). Finally, the D-FROG method was the most robust one to extract and quantify chirp instability, where we observe differences of tens of $\mathrm{fs}^{-2}$ based on different reconstructions of the experimental data. 
As the final step, we characterized pulses generated from a NOPA and measured through a 4fpulse shaper with an SLM-adjusted phase. The SLM phase was adjusted to provide a constant phase, i.e. not to alter the pulse shape. Based on the previous results, we expect the presence of chirp instability and therefore we present only the fit of the experimental data with Equations are (7) and (8) - see Figure 8. By comparing this dataset to the D-FROG traces measured without the SLM (Figure 7), we observed both D-FROG a d-scan experimental traces to be smeared along their horizontal axis, indicating the presence of the chirp instability. By quantifying the chirp instability, the value of $\sigma_{G}$ increased by the SLM involvement from 110 to $240 \mathrm{fs}^{-2}$, which was consistently obtained from the DFROG and d-scan datasets.

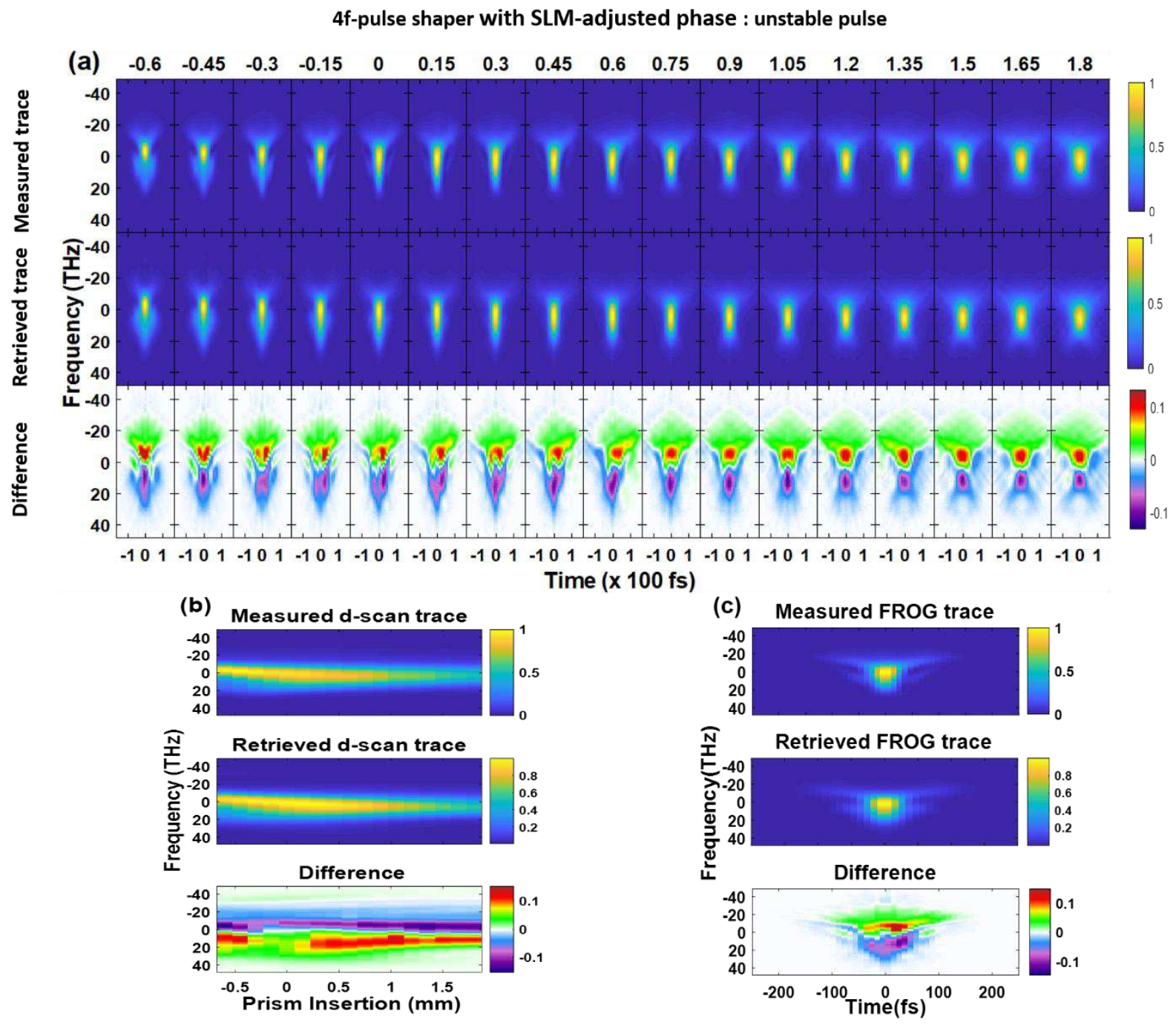


Figure 8. Measured and Retrieved traces of an unstable pulse, generated from NOPA and measured with a 4f-pulse shaper with SLM-adjusted phase, and their differences. (a) D-FROG traces (the traces are acquired for different prism insertion positions from -0.6 to 1.8 , as mentioned above the panels) (b) d-scan trace (c) FROG trace of a compressed pulse. The traces are retrieved using Eq (7) and (8), respectively, where $N=156$ and $d t=1.032 \mathrm{fs}$.

An important question of interest was the source of the increased chirp instability. To understand its origin, we measured a series of FROG traces by using a rapid delay line sweeping, which we converted by spectral integration into the second harmonic generation autocorrelation (SHG-AC) traces. The rapid scan acquisition combined with an increased laser repetition rate (100 kHz) enabled us to scan SHG-AC traces with the acquisition time as short as $60 \mathrm{~ms}$-- see the Supplementary information for details. While we observed a prominent fluctuation of the SHG-AC intensity for the pulses processed by the pulse shaper, we do not observe a significant change in the shape of the SHGAC traces. This holds even when the SLM is used for pulse shaping. Therefore, we conclude that the fluctuating temperature of the SLM elements is likely not the cause of the instability effect $\stackrel{16}{ }$. We can speculate that this effect could be due to the fast fluctuation of the phase, not seen by the rapid scan that we used, or to the spatial chirp caused by the space-time coupling under the effects of the slight misalignment of the PS and the pixelation in the SLM.

\section{Conclusion}

In conclusion, we present a method of dispersion-scan frequency-resolved optical gating (DFROG), which measures FROG traces as a function of dispersion and combines the idea of the FROG technique and the $d$-scan. We used this method to quantify the chirp instability of the ultrafast pulse.

By employing a model that assumes that the quadratic chirp GDD is not a single value but rather a Gaussian distribution, we studied the ability of the FROG, D-FROG and d-scan methods to quantify the chirp instability. We applied the methods on the characterization of pulses generated 
from a NOPA and the same pulses processed by a 4f-pulse shaper, which was used with and without SLM. By extracting the chirp instability for different pulse retrieval approaches, we observed that the D-FROG method provided the most consistent values of chirp instability. This can be understood in terms of the large dataset, which provides a broad consistency check.

Our measurement showed that while the NOPA-generated pulses are stable throughout the measurement, the chirp instability appears for the pulses processed by the pulse shaper (approx. 110 $\left.\mathrm{fs}^{-2}\right)$ and it becomes larger with the use of SLM $\left(240 \mathrm{fs}^{-2}\right)$. However, our rapid-scan measurements, where a single FROG trace was acquired within $60 \mathrm{~ms}$, did not show any prominent variation in the pulse shape. Therefore, we ascribe this effect to a combination of the fast fluctuation of the phase and to the space-time coupling induced by a misalignment of the pulse shaper.

Our result shows that the pulse instability reaching $100-200 \mathrm{fs}^{-2}$ can be present even in the cases, where a single FROG trace of a $20 \mathrm{fs}$ pulse can be reliably reproduced with a realistic pulse shape. In such cases, the D-FROG method provides a way to identify and quantify the instability level.

\section{Acknowledgment}

The authors gratefully acknowledge the financial support from The Czech Academy of Sciences (ERCCZ/AV-B, Project Random-phase Ultrafast Spectroscopy; ERC100431901), and the Ministry of Education, Youth and Sports ("Partnership for Excellence in Superprecise Optics," Reg. No. CZ.02.1.01/0.0/0.0/16_026/0008390).

\section{Reference}

1. P. Balling and J. Schou. Femtosecond-laser ablation dynamics of dielectrics: basics and applications for thin films. Rep. Prog. Phys. 76, 036502 (2013).

2. W. ShuFeng, G. QiHuang. Progress in femtochemistry and femtobiology. Science China Physics, Mechanics and Astronomy. 54, 2103-2108 (2011). 
3. X. Wang and N. Wada. Spectral phase encoding of ultra-short optical pulse in time domain for OCDMA application. Opt. Express. 12, 7320 (2007).

4. J. Paye, M. Ramaswamy, J. G. Fujimoto, and E. P. Ippen. Measurement of the amplitude and phase of ultrashort light pulses from spectrally resolved autocorrelation. OPTICS LETTERS. 18, 22 (1993).

5. Bern Kohler, Vladislav V. Yakovlev, and Kent R. Wilson. Phase and intensity characterization of femtosecond pulses from a chirped-pulse amplifier by frequency-resolved optical gating. Opt. Lett. 20, 5 (1995).

6. Rana Jafari and Rick Trebino, Member, IEEE. Highly Reliable Frequency-Resolved Optical Gating Pulse-Retrieval Algorithmic Approach. IEEE JOURNAL OF QUANTUM ELECTRONICS. 55, 4 (2019).

7. Y. Kida, Y. Nakano, K. Motoyoshi,and T. Imasaka. Frequency-resolved optical gating with two nonlinear optical processes. Opt. Lett . 39, 10 (2014).

8. C. laconis and I. A. Walmsley. Spectral phase interferometry for direct electric-field reconstruction of ultrashort optical pulses. Opt. Lett. 23, 792-794 (1998).

9. V. V. Lozovoy, I. Pastirk, and M. Dantus. Multiphoton intrapulse interference IV Ultrashort laser pulse spectral phase characterization and compensation. Opt. Lett. 29, 775-777 (2004).

10. F. Silva, M. Miranda, B. Alonso, J. Rauschenberger, V. Pervak, H. Crespo. Simultaneous compression, characterization and phase stabilization of GW-level 1.4 cycle VIS-NIR femtosecond pulses using a single dispersion-scan setup. 22, 10181-10190 (2014).

11. J. Ratner, G.Steinmeyer, T. Chun Wong, R. Bartels, and Rick Trebino. Coherent artifact in modern pulse measurements. Opt. Lett. 37, 2874-2876 (2012).

12. E. Escoto, R. Jafari, G. Steinmeyer, R.Trebino. Linear chirp instability analysis for ultrafast pulse metrology. Journal of the Optical Society of America B 37, 74-81 (2020). 
13. M. Rhodes, Z. Guang and R. Trebino. Unstable and Multiple Pulsing Can Be Invisible to Ultrashort Pulse Measurement Techniques. Appl. Sci. 7 (2017).

14. F. Frei, A. Galler, and T. Feurer. Space-time coupling in femtosecond pulse shaping and its effects on coherent control. THE JOURNAL OF CHEMICAL PHYSICS. 130, 034302 (2009).

15. B. J. Sussman, R. Lausten, and A. Stolow. Focusing of light following a 4-f pulse shaper: Considerations for quantum control. PHYSICAL REVIEW A. 77, 043416 (2008).

16. E. BRÜHL, T. BUCKUP, AND M. MOTZKUS. Minimization of $1 / \mathrm{fn}$ phase noise in liquid crystal masks for reliable femtosecond pulse shaping. Opt. Express. 25, 19 (2017).

17. B. Döpke, J. C. Balzer and M. R. Hofmann. Phase and amplitude calibration of dual mask spatial light modulator for highresolution femtosecond pulse shaping. Electronics Letters. 51, 642-644 ( 2015).

18. Y. Calderon-Hermosillo, J. Garcia-Marquez, R. Espinosa-Luna, N. A. Ochoa, V. Lopez, A. Aguilar, E. Noe'-Arias, Y. Alayli. Flicker in a twisted nematic spatial light modulator. Optics and Lasers in Engineering. 51, 741-748 (2013).

19. B. Alonso, S. Torres-Peiró, R. Romero, P. T. Guerreiro, A. Almagro-Ruiz, H. Muñoz-Marco, P. PérezMillán \& H. Crespo. Detection and elimination of pulse train instabilities in broadband fibre lasers using dispersion scan. Scientific Reports. 10, 7242 (2020).

20. B. Alonso, Í. J. Sola \& H. Crespo. Self-calibrating d-scan: measuring ultrashort laser pulses ontarget using an arbitrary pulse compressor. Scientific Reports. 8, 3264 (2018).

21. P. Sidorenko, O. Lahav, Z. Avnat, and O. Cohen. Ptychographic reconstruction algorithm for frequency-resolved optical gating: super-resolution and supreme robustness. Optic. 3, 1320-1330 (2016). 
Figures
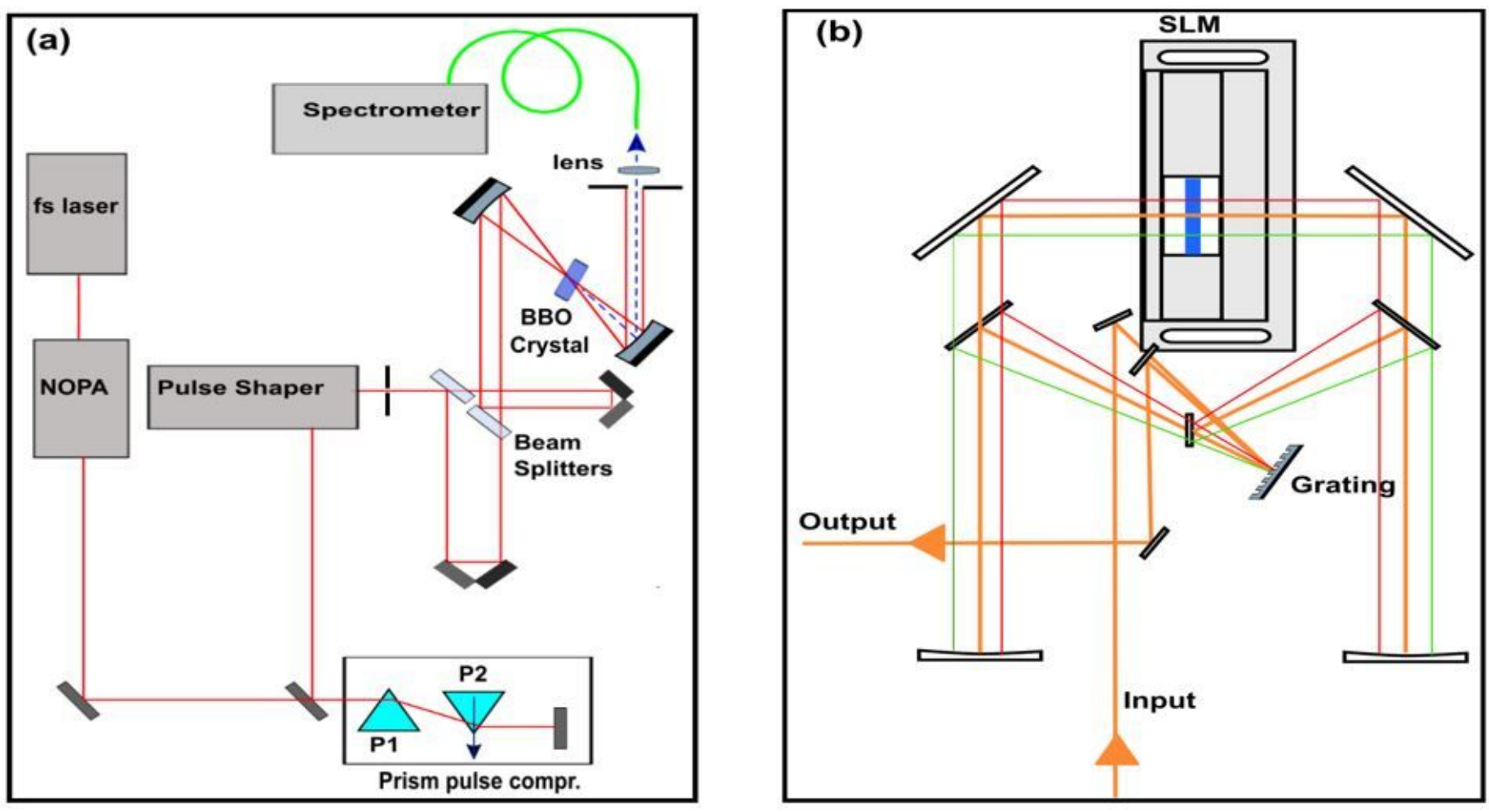

Figure 1

(a) Experimental setup for the D-FROG. (b) Pulse shaper in $4 f$ geometry. 

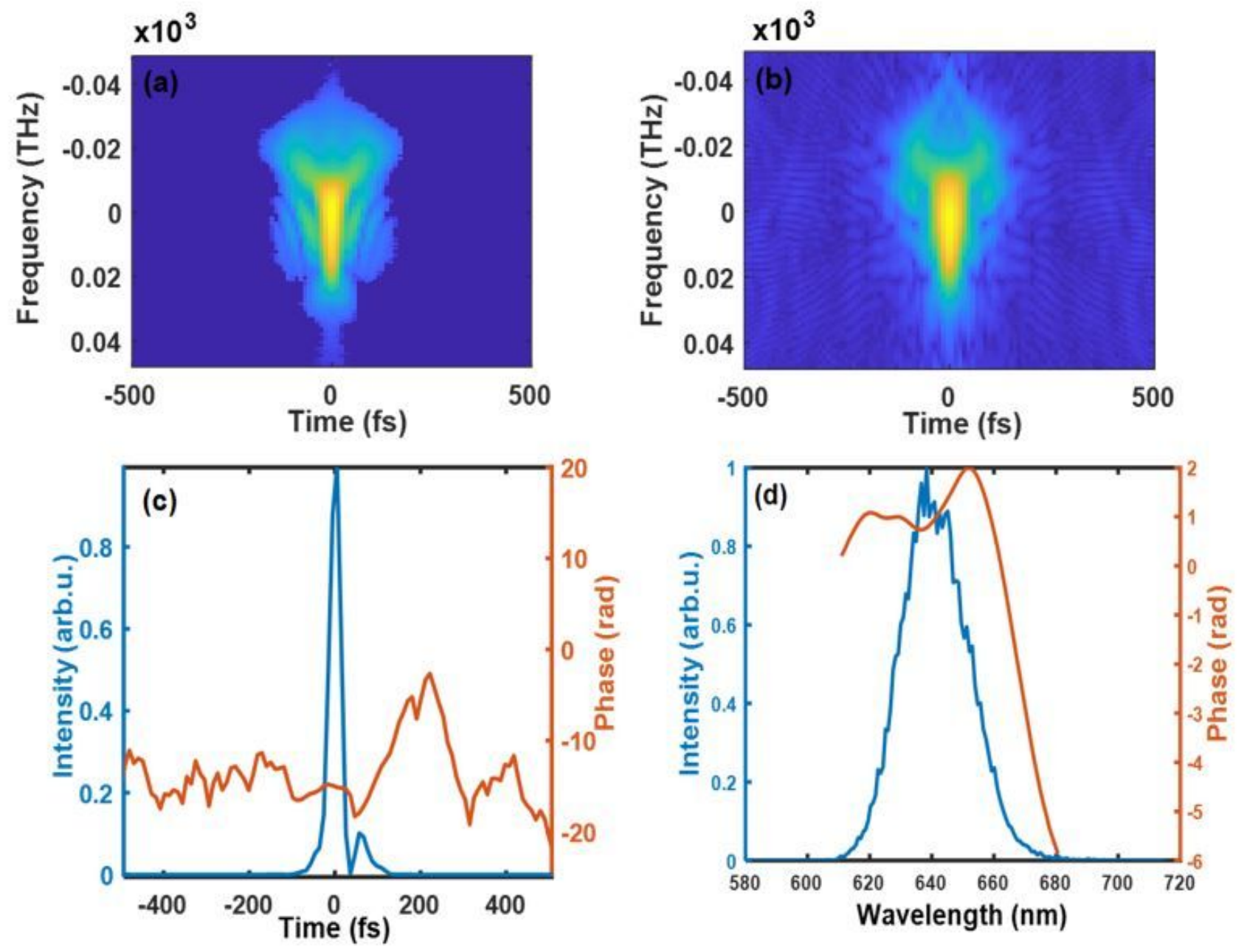

Figure 2

Measured (a) and Retrieved (b) FROG traces from a compressed NOPA pulse. (c) Retrieved temporal intensity profiles and phases. (d) Retrieved spectral intensity profiles and phases. $\mathrm{N}=156, \mathrm{dt}=1.032 \mathrm{fs}$. The retrieved fields with rms widths 16.75 fs. 


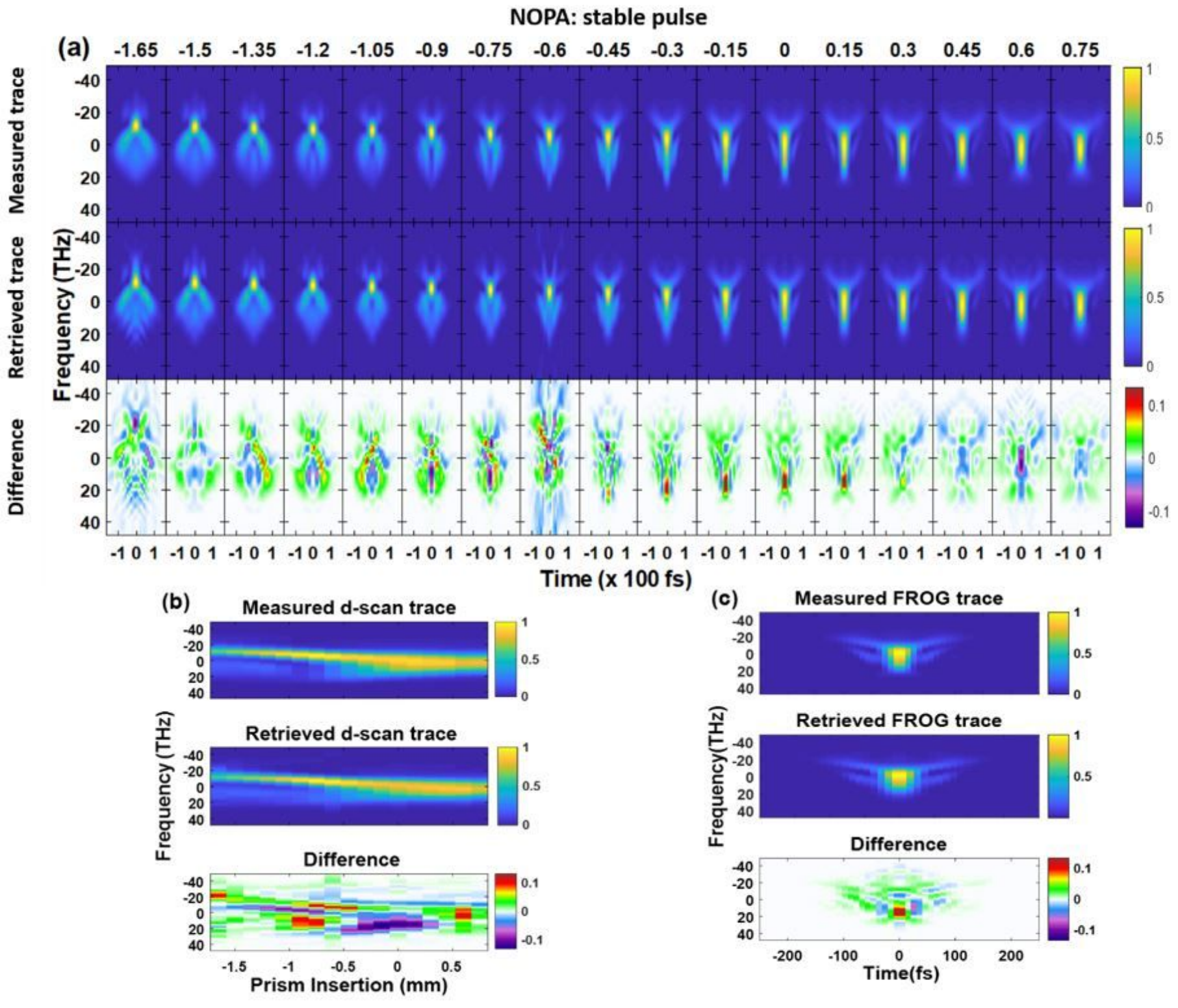

Figure 3

Measured and Retrieved traces of an ideal stable pulse, generated from NOPA, and their differences. (a) D-FROG traces (the traces are acquired for different prism insertion positions from -1.65 to 0.75 , as mentioned above the panels) (b) d-scan trace (c) FROG trace of a compressed pulse. The traces are retrieved using Eq (1) and (2), respectively, where $\mathrm{N}=156$ and $\mathrm{dt}=1.032 \mathrm{fs}$. 
NOPA: unstable pulse
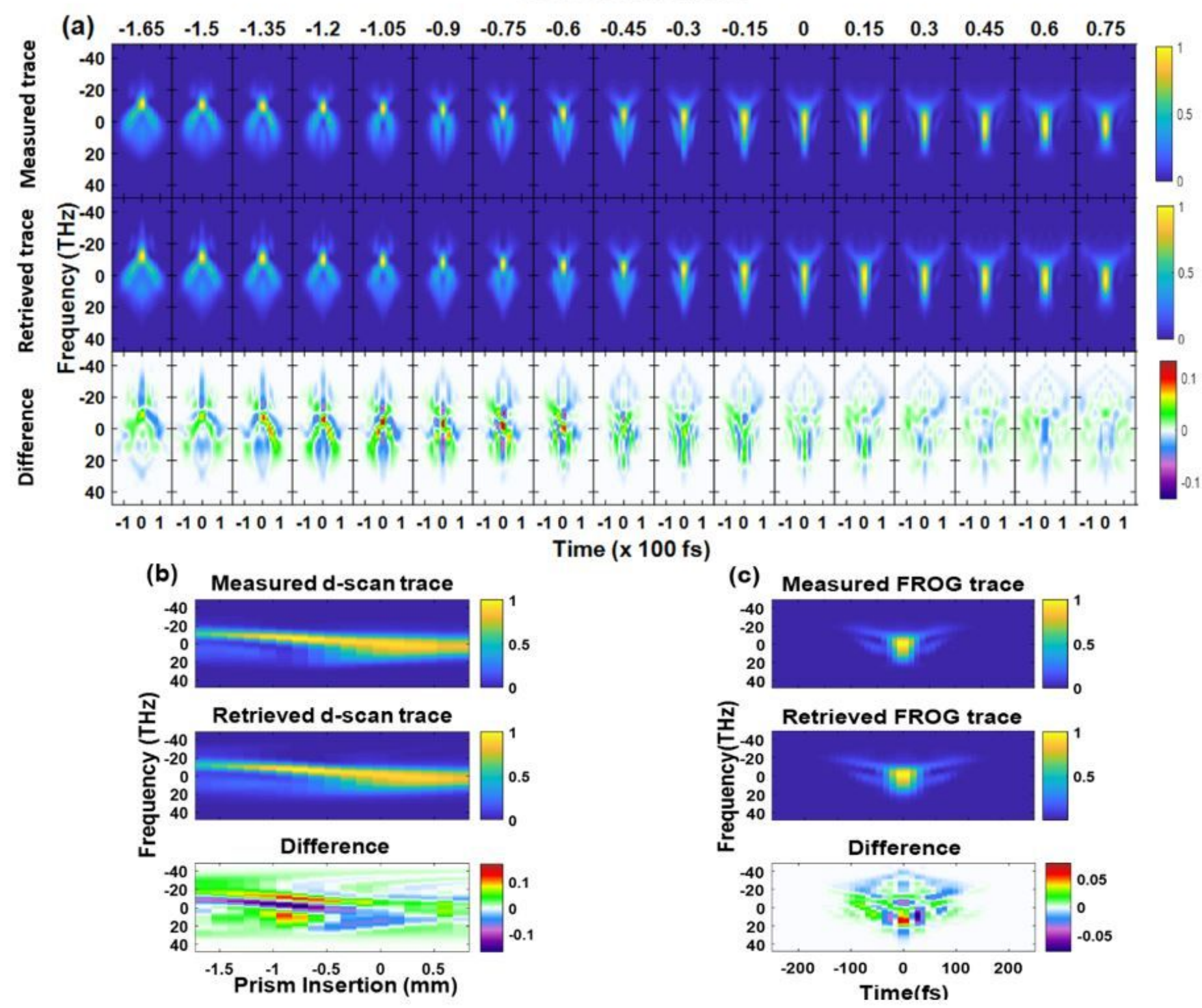

Figure 4

Measured and Retrieved traces of an unstable pulse, generated from NOPA, and their differences. (a) DFROG traces (the traces are acquired for different prism insertion positions from -1.65 to 0.75 , as mentioned above the panels) (b) d-scan trace (c) FROG trace of a compressed pulse. The traces are retrieved using Eq (7) and (8), respectively, where $\mathrm{N}=156$ and $\mathrm{dt}=1.032 \mathrm{fs}$. 

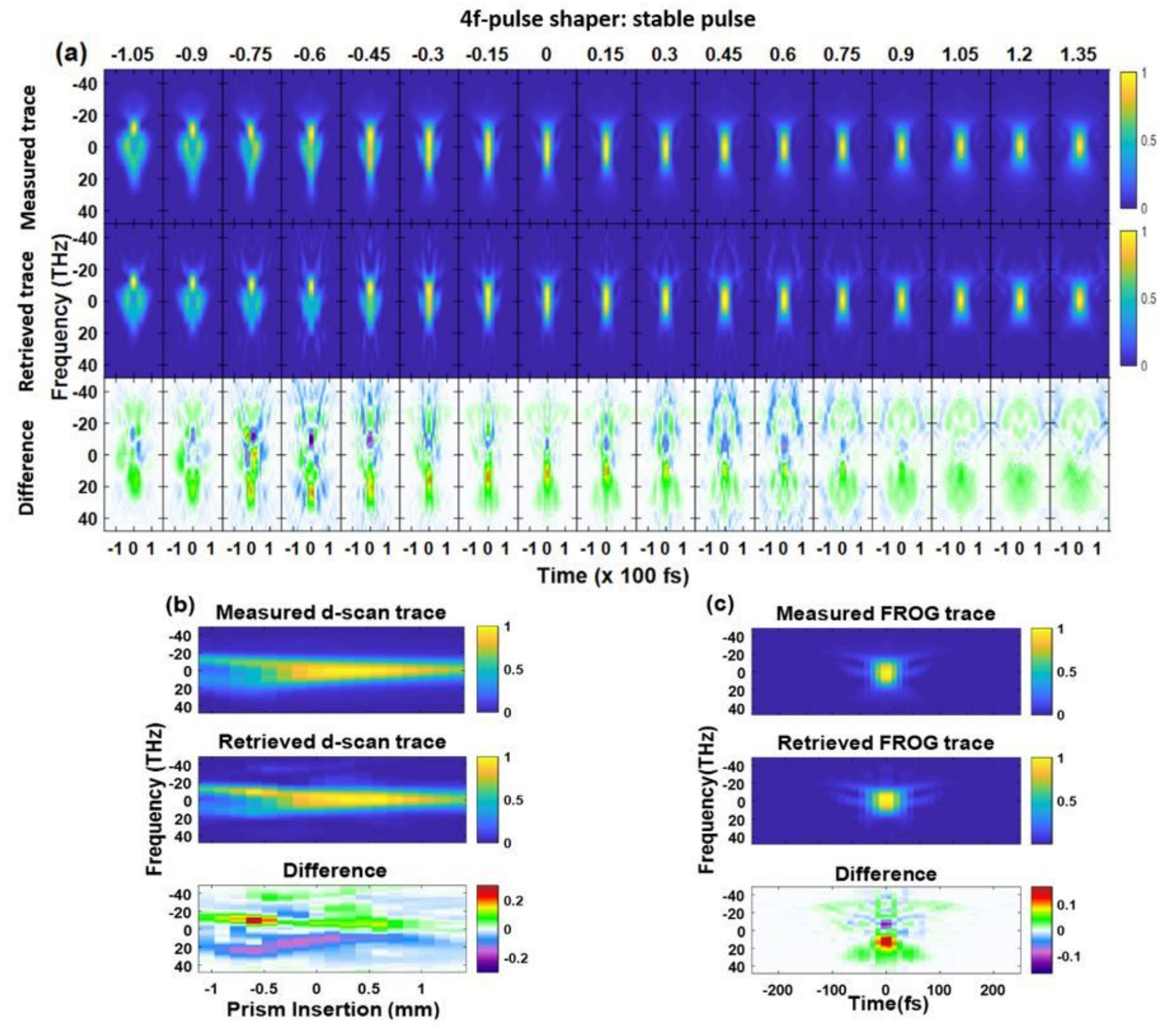

\section{Figure 5}

Measured and Retrieved traces of an ideal stable pulse, generated from NOPA and measured with a 4fpulse shaper, and their differences. (a) D-FROG traces (the traces are acquired for different prism insertion positions from -1.05 to 1.35, as mentioned above the panels) (b) d-scan trace (c) FROG trace of a compressed pulse. The traces are retrieved using Eq (1) and (2), respectively, where $\mathrm{N}=156$ and dt=1.032 fs. 


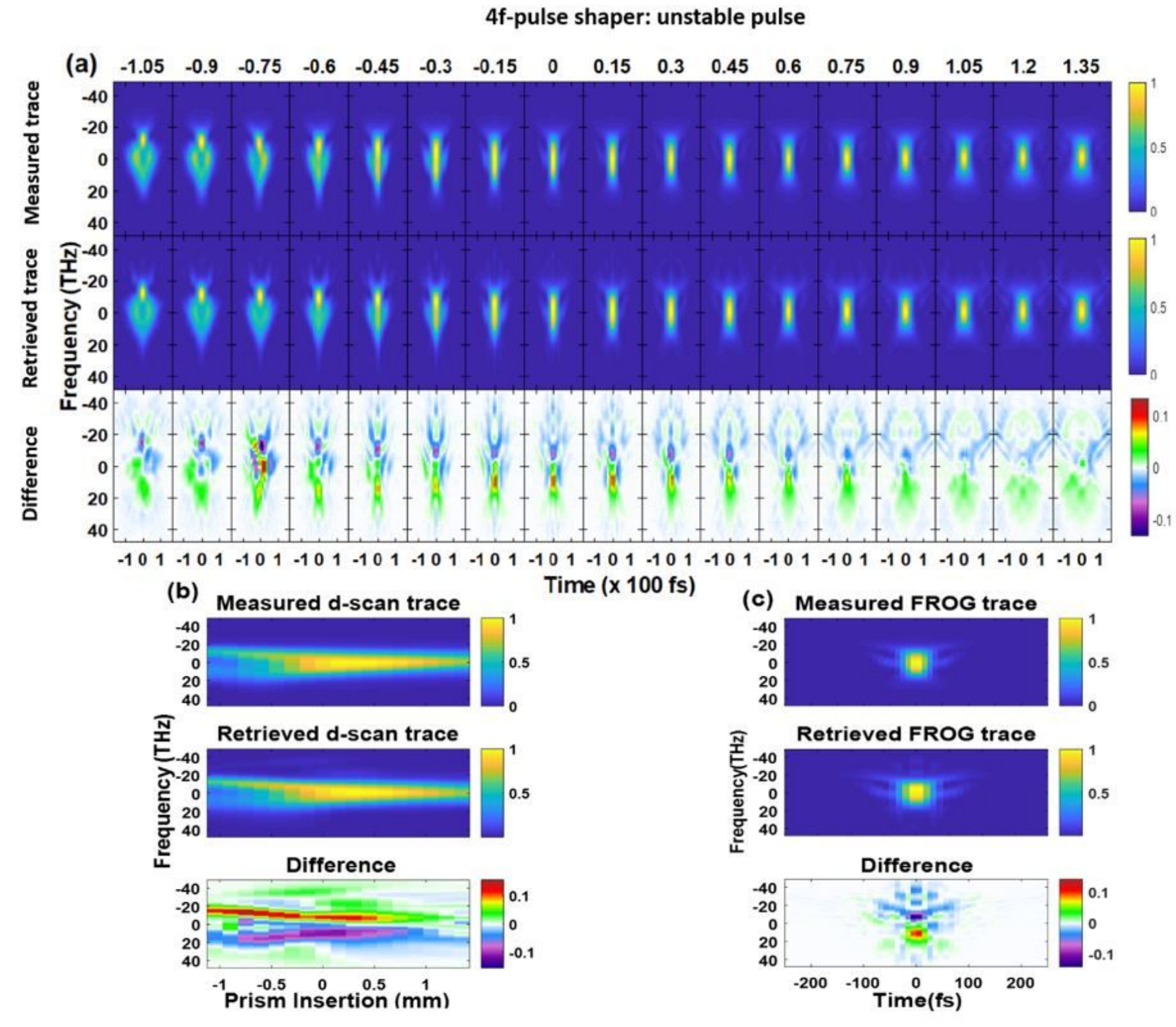

Figure 6

Measured and Retrieved traces of an unstable pulse, generated from NOPA and measured with a 4f-pulse shaper, and their differences. (a) D-FROG traces (the traces are acquired for different prism insertion positions from -1.05 to 1.35, as mentioned above the panels) (b) d-scan trace (c) FROG trace of a compressed pulse. The traces are retrieved using Eq (7) and (8), respectively, where $\mathrm{N}=156$ and $\mathrm{dt}=1.032$ fs. 

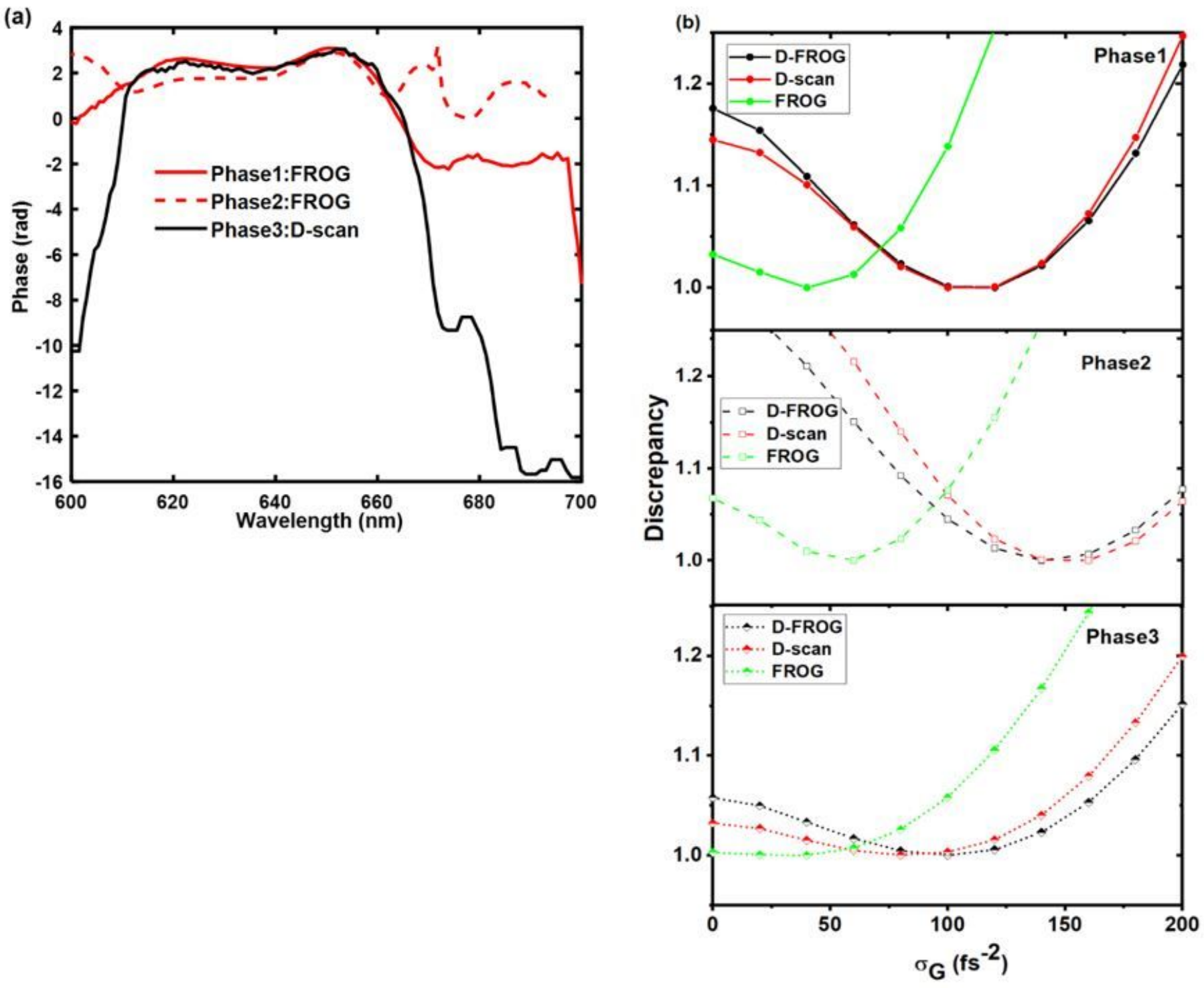

Figure 7

(a) Spectral phases, retrieved using FROG and d-scan methods, of a pulse generated from a NOPA and measured through a $4 \mathrm{f}$-pulse shaper without any modulation device. (b) Discrepancy versus the chirp instability $\sigma \_G$, using three different phases. 
4f-pulse shaper with SLM-adjusted phase : unstable pulse

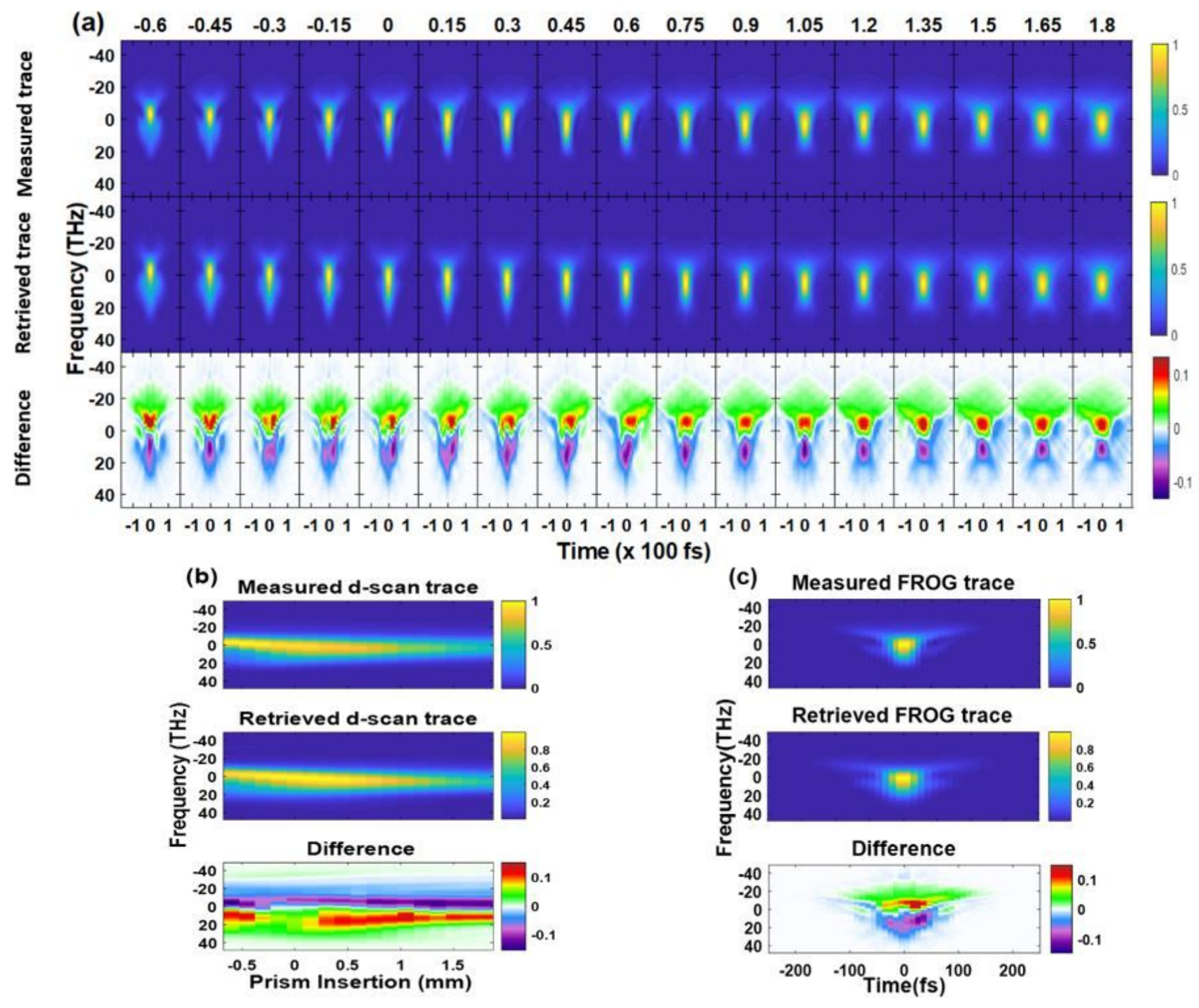

Figure 8

Measured and Retrieved traces of an unstable pulse, generated from NOPA and measured with a 4f-pulse shaper with SLM-adjusted phase, and their differences. (a) D-FROG traces (the traces are acquired for different prism insertion positions from -0.6 to 1.8 , as mentioned above the panels) (b) d-scan trace (c) FROG trace of a compressed pulse. The traces are retrieved using Eq (7) and (8), respectively, where $\mathrm{N}=156$ and $\mathrm{dt}=1.032 \mathrm{fs}$.

\section{Supplementary Files}

This is a list of supplementary files associated with this preprint. Click to download.

- Supplementaryinformation.docx 